\title{
Velocity dispersion around ellipticals in MOND
}

\author{
O. Tiret ${ }^{1}$, F. Combes ${ }^{1}$, G. W. Angus ${ }^{2}$, B. Famaey ${ }^{3}$, and H. S. Zhao ${ }^{2}$ \\ 1 Observatoire de Paris, LERMA, 61 Av. de l'Observatoire, 75014 Paris, France \\ e-mail: olivier.tiret@obspm.fr \\ 2 SUPA, School of Physics and Astronomy, Univ. of St. Andrews, Scotland KY169SS, UK \\ 3 Institut d'Astronomie et d'Astrophysique, Univ. Libre de Bruxelles, CP 226, Bvd du Triomphe, 1050 Bruxelles, Belgium
}

Received 29 August 2007 / Accepted 15 October 2007

\section{ABSTRACT}

\begin{abstract}
We investigate how different models that have been proposed for solving the dark matter problem can fit the velocity dispersion observed around elliptical galaxies, on either a small scale $(\sim 20 \mathrm{kpc})$ with stellar tracers, such as planetary nebulae, or large scale $(\sim 200 \mathrm{kpc})$ with satellite galaxies as tracers. Predictions of Newtonian gravity, either containing pure baryonic matter, or embedded in massive cold dark matter (CDM) haloes, are compared with predictions of the modified gravity of MOND. The standard CDM model has problems on a small scale, and the Newtonian pure baryonic model has difficulties on a large scale, while a fit with MOND is possible on both scales.
\end{abstract}

Key words. galaxies: elliptical and lenticular, $\mathrm{cD}$ - galaxies: kinematics and dynamics - cosmology: dark matter

\section{Introduction}

Measuring the velocity field in and around galaxies is the main way to test the dark matter distribution at small and intermediate scales. The observation of what are apparently non-Newtonian rotation curves around spiral galaxies (e.g. Rubin et al. 1980) has been first solved by assuming that galaxies are embedded in dark matter haloes. Numerical simulations, however, predict a radial distribution for the CDM model much more concentrated than what is observed (Gentile et al. 2004; de Blok 2005).

An alternative explanation was proposed by Milgrom (1983), as MOdified Newtonian Dynamics (MOND). When the Newtonian acceleration falls below the critical value $a_{0} \sim 2 \times$ $10^{-10} \mathrm{~m} \mathrm{~s}^{-2}$, the gravity law is empirically modified and then declines in $1 / r$ instead of $1 / r^{2}$. Around spherical systems, the modified acceleration $g$ satisfies the relation

$g \mu\left(g / a_{0}\right)=g_{\mathrm{n}}$

where $g_{\mathrm{n}}$ is the Newtonian acceleration. For non-spherical geometry, this is only an approximation. However, in this Letter we only consider spherical systems as representing elliptical galaxies and we adopt $\mu(x)=x / \sqrt{1+x^{2}}$.

This model is very successful on a galactic scale; in particular, it explains a large number of rotation curves of galaxies, with some exceptions (Gentile et al. 2004), and naturally the Tully-Fisher relation, (e.g. Sanders \& McGaugh 2002). While the dark matter problem is observationally very clear around spiral galaxies, thanks to their rotation curve measured with the cold hydrogen gas at $21 \mathrm{~cm}$, which is nearly in circular orbits (e.g. Bosma 1981; Verheijen \& Sancisi 2001), the situation is much more complex around elliptical galaxies, with little or no rotation.

Recently, planetary nebulae have been used as an efficient tool for measuring the velocity field at large radii in early-type galaxies, and they complement stellar absorption kinematical studies (Romanowky et al. 2003). In typical elliptical galaxies, the velocity-dispersion profiles were found to decline with radius, up to 5 effective radii, thereby requiring no dark matter at all. Dekel et al. (2005) show that the data are still compatible with the usual dark matter models, if the planetary nebulae tracers have particularly radial orbits in the outer parts, because of a recent merger with small impact parameter. However, the recent results from Douglas et al. (2007) challenge this interpretation, prolonging the decline to more than 7 effective radii.

On larger scales around early-type galaxies, from 50 to $300 \mathrm{kpc}$, Klypin \& Prada (2007), KP07, have proposed to test gravity models with satellite galaxies as a tracer. From the Sloan Digital Sky Survey, they stack several thousand galaxies in 3 luminosity classes and determine the number density of satellites and their velocity dispersion around them. In each mass range, the radial distributions are obtained with around 1500 satellites, although about 1.5 satellite exist around each galaxy.

This large-scale galaxy neighborhood has not been widely tested yet in modified gravity. The well-known difficulty of MOND in clusters has found a possible solution with neutrinos of $2 \mathrm{eV}$ mass (Sanders 2003; Angus et al. 2007a; but see Zhao 2007), and the escape velocity around giant galaxies like the Milky Way was shown to correspond to observations, when including the external field effect (Famaey et al. 2007; Wu et al. 2007).

In this work, we solve the Jeans equation for the distribution of the velocity dispersion around elliptical galaxies, and in particular we fit the NGC 3379 galaxy, where the most extended data is available for the velocities. We also further explore fits at larger radii for the special case of NGC 3379, with satellite galaxies as tracers, as done by KP07 and Angus et al. (2007b). This is only statistically valid around a generic 


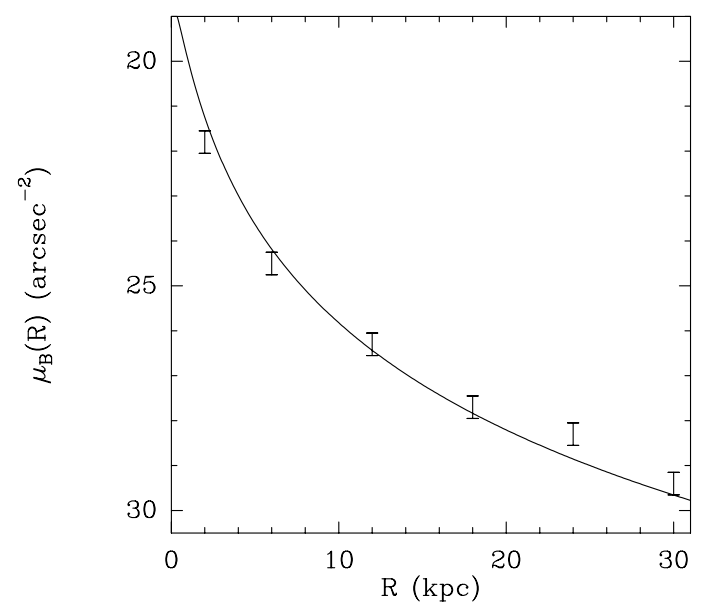

Fig. 1. Fit of the blue-light distribution in NGC 3379. The data points are taken from Douglas et al. (2007). The curve represents the light profile derived with the mass-to-light ratio given in Table 1 and a mass model of cored-Hernquist type (Table 2).

Table 1. Parameters for deriving the mass of NGC 3379.

\begin{tabular}{cccc}
\hline \hline$D(\mathrm{Mpc})$ & $M_{B}$ & $B_{T c}$ & $M / L_{B}$ \\
\hline 9.8 & -19.9 & 10.10 & 7.0 \\
\hline
\end{tabular}

Table 2. Radial distribution of the tracer density.

\begin{tabular}{ccccc}
\hline \hline & $\alpha_{0}$ & $\alpha_{1}$ & $r_{\alpha}$ & $\epsilon$ \\
\hline stars \& PN & -1 & -3 & 1.1 & 1.2 \\
Sat & -1.7 & -1.5 & 150 & 0 \\
\hline$\rho(r) \propto(r+\epsilon)^{\alpha_{0}}\left(r+r_{\alpha}\right)^{\alpha_{1}} ; \epsilon, r_{\alpha}$ in kpc.
\end{tabular}

early-type galaxy, with a mass comparable to NGC 3379. This is the brightest galaxy of a group, but the observed companion velocity is not statistically significant.

\section{The Jeans equation}

Because of the spherical symmetry, it has been shown (Angus et al. 2007) that the Jeans equation can be written in MOND, as well as in DM:

$\frac{\mathrm{d} \sigma^{2}}{\mathrm{~d} r}+\sigma^{2} \frac{(2 \beta+\alpha)}{r}=-g(r)$

where $\sigma$ is the radial velocity dispersion, $\alpha=\mathrm{d} \ln \rho / \mathrm{d} \ln r$ is the slope of the tracer density $\rho$, and $\beta=1-\left(\sigma_{\theta}^{2}+\sigma_{\phi}^{2}\right) / 2 \sigma^{2}$ is the velocity anisotropy.

The Jeans equation is usually used for a unique selfgravitating component, where the density $\rho$ appearing in the "pressure" term on the left is the same as the density appearing in the Poisson equation, giving the density field $g(r)$ on the right. On the left side, we use the density and the velocity dispersion of the tracers only, which can be very different from the density producing the potential, in particular for the satellite galaxies, which act as test particles.

In the present approach, we want to compare all models (Newtonian, with or without CDM and MOND) with the same dynamics for the Jeans equation, fitting the density of tracers as close as possible to the observed density distribution. For the CDM models, we consider two different amounts of dark matter: (i) the CDM1 model, reminiscent of what is found in cosmological simulations, where the amount of dark matter inside the radius of $200 \mathrm{kpc}$ is equal to 60 times the visible matter; (ii) the CDM2 model, more akin to what is required to model rotation curves of spiral galaxies: the amount of dark matter inside the radius of $200 \mathrm{kpc}$ is equal to 20 times the visible matter.

All models are then compared with the same tools, while KP07 compare the CDM model with numerical simulations, and MOND with the Jeans equation, but without exploring all variations of the tracer density and anisotropy profiles.

\section{Results}

NGC 3379 is modeled by a cored-Hernquist distribution (see Fig. 1). The stellar mass of this galaxy is estimated to be $M=$ $1 \times 10^{11} M_{\odot}$, assuming a constant mass-to-light ratio $\mathrm{M} / \mathrm{L}$ (see Table 1). In MOND (and Newton without DM), this stellar distribution is the only matter contributing to the gravitational potential. In Newtonian dynamics with DM, the DM halo follows the NFW radial profile found in $N$-body cosmological simulations. We used three kinds of tracers to compare Newton without DM, CDM1, CDM2, and MOND models, for different length scales: stars for the inner part ( $r<1 \mathrm{kpc}$, Shapiro et al. 2006), planetary nebulae for the middle part $(1 \mathrm{kpc}<r<20 \mathrm{kpc}$, Douglas et al. 2007), and satellites (20 kpc $<r<200 \mathrm{kpc}, \mathrm{KP} 07)$. The satellites do not correspond to the real ones orbiting NGC 3379, but are a statistical representation from the SDSS (KP07), giving the velocity dispersion of satellites submitted to the gravity of a typical elliptical galaxy in the same mass category as NGC 3379. The parameters of the tracer density are displayed in Table 2.

We numerically solve the Jeans equation for each tracer. The projected velocity dispersion $\left(\sigma_{\text {los }}\right)$ is represented in Fig. 2.

\subsection{Newton dynamics without DM}

- Stars: the projected velocity dispersion $\left(\sigma_{\text {los }}^{\star}\right)$ is well-fitted using a small radial anisotropy increasing with radius from $\beta=0.2$ to $\beta=0.4$. Since the acceleration here is above the critical $a_{0}$, both MOND and Newton share the same fitting parameters.

- PN: as for the stars, the observed PN velocity dispersion $\left(\sigma_{\text {los }}^{\mathrm{PN}}\right)$ is also in good agreement with the Newtonian model. It implies a tangential velocity in the central region $(\beta=-0.6)$ evolving to radial trajectories $(\beta=0.8)$.

- Satellites: $\sigma_{\text {los }}^{\text {sat }}$ cannot be fitted at all by considering the stellar mass alone. A dark matter component is required.

\subsection{CDM1: DM halo mass from cosmological simulations}

The mass of the NFW halo is 60 times the stellar mass inside $200 \mathrm{kpc}$.

- Stars: the cusp of the DM halo does not allow fitting the $\sigma_{\text {los }}^{\star}$, whatever the anisotropy profile. The predicted velocity dispersion is too large.

- PN: $\sigma_{\text {los }}^{\mathrm{PN}}$ does not fit the entire data set. The dispersion is too large around $10 \mathrm{kpc}$. If $\beta$ is increased towards its maximum, up to a purely radial anisotropy profile (which tends to decrease the velocity dispersion), $\sigma_{\text {los }}^{\mathrm{PN}}$ is then too small in the inner part. In the best fit, the value of $\beta$ is maximum at $1 \mathrm{kpc}$.

- Satellites: the model reproduces the observations well, if radial anisotropy is maintained all over the region $(\beta>0.8)$. 

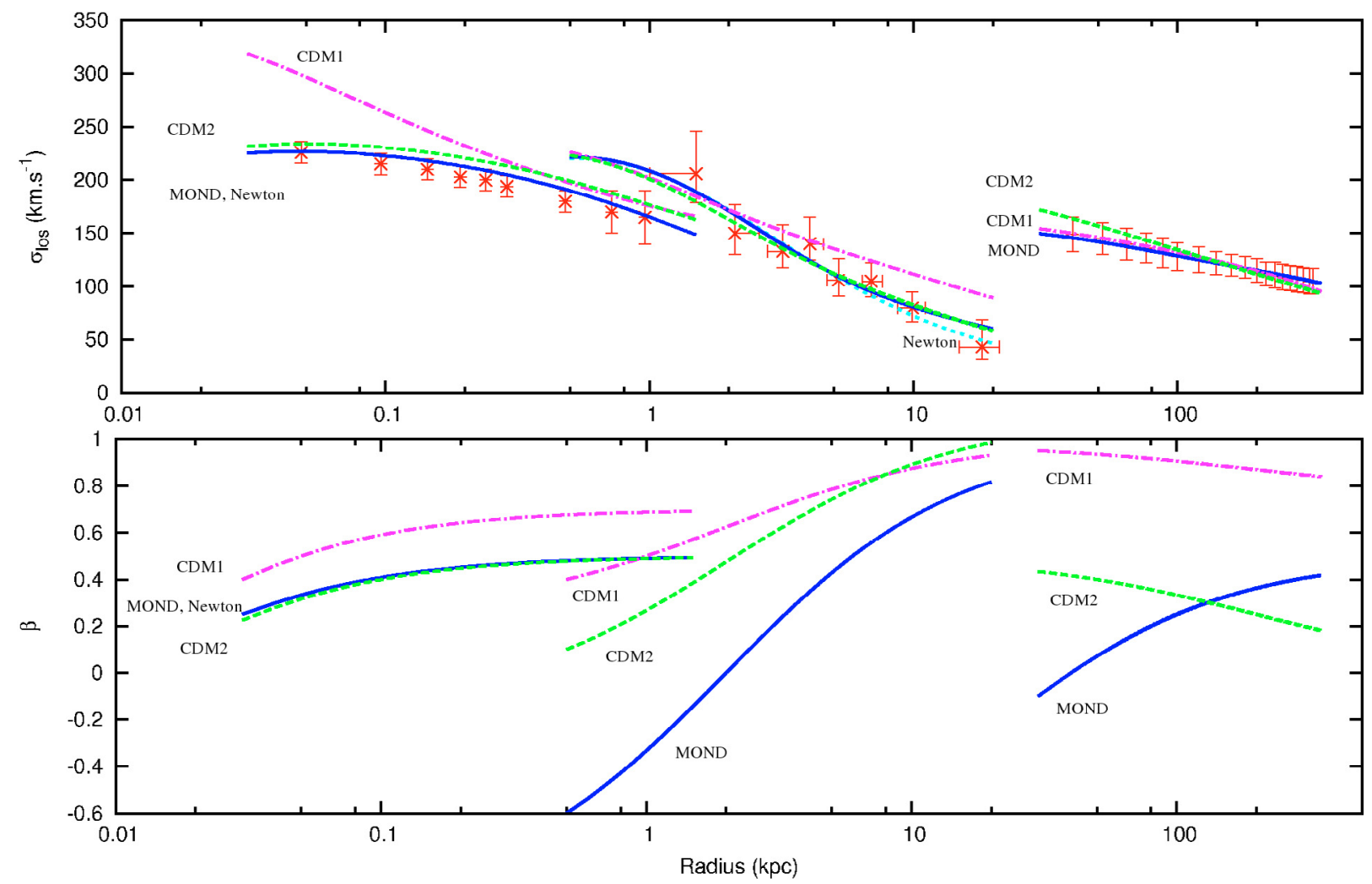

Fig. 2. Fit of the velocity dispersion data around NGC 3379 with pure a Newton model without DM, CDM1, CDM2, and MOND. The data points in the very center $(r<1 \mathrm{kpc})$ are from Shapiro et al. (2006). On intermediate scales they are taken from Douglas et al. (2007) and are based around $10-30 \mathrm{kpc}$ on the planetary nebulae as tracers. On large scales, the data points correspond to the statistical distribution from the satellite galaxies as tracers (KP07), adapting NGC 3379 in their small luminosity class. The tracer density is taken as an Hernquist distribution on a small scale, and fitted to the satellite density distribution on a large-scale. The best fit is obtained with varying $\beta(r)$. Purple/dot-dash: CDM1. Green/dash: CDM2. Blue/full line: MOND, Cyan/short dash: Newton without DM.

\subsection{CDM2: DM halo mass compatible with average spirals}

The mass of the NFW halo is 20 times the stellar mass inside $200 \mathrm{kpc}$.

- Stars \& PN: they are well-fitted, and the DM halo cusp is no longer a problem in the internal dynamics of the galaxy. For the PN, the velocity dispersion is isotropic near the center $(r<1 \mathrm{kpc})$ and increase to its maximum $\beta=1$ at $20 \mathrm{kpc}$.

- Satellites: it is more difficult to fit $\sigma_{\text {los }}^{\text {sat }}$ with less DM. We need to impose an isotropic velocity dispersion $(\beta \sim 0)$, to avoid too fast 0 a fall in $\sigma_{\text {los }}^{\text {sat }}$. The negative gradient of $\beta(r)$ helps to straighten the slope of $\sigma_{\text {los }}^{\text {sat }}(r)$ which otherwise is too steep.

We also varied the dark-to-visible mass ratio between 10 to 60 , and 20 gives the best compromise between the $1-10 \mathrm{kpc}$ and 30-200 kpc regions.

\subsection{MOND}

All scales are easy to fit with MOND.

- Stars: under $1 \mathrm{kpc}$, the gravitational potential is purely Newtonian, so MOND and Newton models are identical. The anisotropy needs to be a bit radial $(\beta \sim 0.2-0.4)$, but always far from the allowed limits.

- PN: until $5 \mathrm{kpc}$ the potential is still Newtonian. A slight difference appears after $8 \mathrm{kpc}$. When varying $\beta(r)$ from -0.6 (tangential) to 0.8 (radial), the MOND model is in good agreement with the observations.
Table 3. Best fit for the anisotropy distributions.

\begin{tabular}{cccccc}
\hline \hline & & MOND & CDM1 & CDM2 & Newton \\
\hline Stars & $r_{\beta}=$ & 0.01 & 0.01 & 0.01 & 0.01 \\
& $\beta_{0}=$ & -0.5 & -0.5 & -0.6 & -0.5 \\
& $\beta_{1}=$ & 1 & 1.2 & 1.1 & 1. \\
\hline PN & $r_{\beta}=$ & 2 & 2 & 2 & 2 \\
& $\beta_{0}=$ & -1 & 0.25 & -0.15 & -1 \\
& $\beta_{1}=$ & 2 & 0.75 & 1.25 & 1.8 \\
\hline Sat & $r_{\beta}=$ & 20 & 200 & 200 & - \\
& $\beta_{0}=$ & -1 & 0.98 & -0.5 & - \\
& $\beta_{1}=$ & 1.5 & -0.22 & 0.5 & - \\
\hline
\end{tabular}

$\beta(r)=\beta_{0}+\beta_{1} r /\left(r+r_{\beta}\right) ; r_{\beta}$ in kpc.

- Satellites: $\sigma_{\text {los }}^{\text {sat }}$ is also well-fitted by MOND on this scale, using $\beta=-0.2$ at $r=20 \mathrm{kpc}$ to $\beta=0.2$ at $r=200 \mathrm{kpc}$.

Figure 3 shows how the variations in $\beta$ can help to fit many versions of the velocity curves. As in all other models, there is still much latitude to the fit. Large spatial variations of $\beta$ are possible if the ellipticals are the results of mergers (Dekel et al. 2005)

\section{Discussion}

In contrast to the conclusion of KP07, MOND does not predict constant velocity dispersion with radius in the neighborhood of elliptical galaxies. There is a wide latitude for varying the possible anisotropy parameter according to the scale and the tracer considered. Our best fit starts from a tangential or isotropic configuration near the center and evolves progressively to a radial 


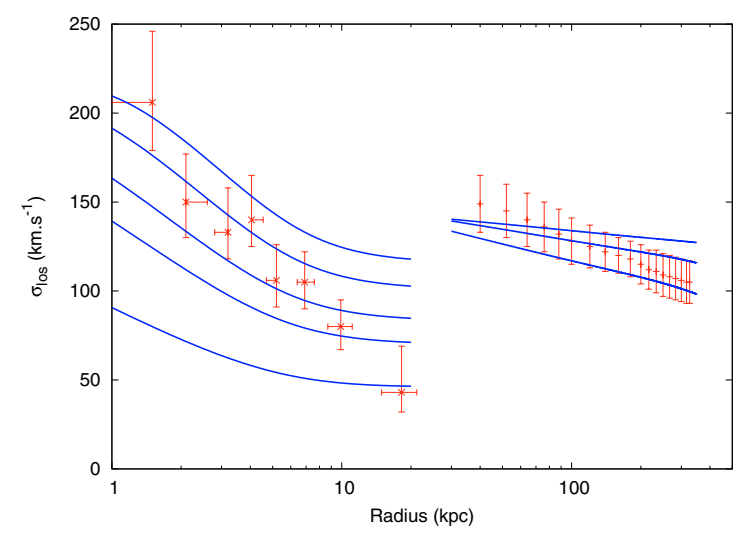

Fig. 3. Fit of the velocity dispersion data around NGC 3379 with MOND, and various values of $\beta$. The data points are the same as in Fig. 2. The 4 curves on a small-scale are $\beta=-1,0,0.5,0.9$; and $\beta=$ $-1,0,0.5$ on a large-scale.

one for each of the three scales considered, which appears quite realistic.

While the Newtonian model without dark matter has problems in the outer parts, the CDM1 model encounters severe difficulties in the inner parts. The CDM2 model, with a reduced dark matter relative to the visible mass, can also fit the data quite well. Compared to the MOND model, it requires a larger radial anisotropy on each scale, and its $\beta$ profile is unusual for the satellite tracer with a negative slope. For $\beta=$ const., the $\sigma_{\text {los }}^{\text {sat }}$ would be too steep compared to the observations. And by introducing anisotropy increasing with radius, the $\sigma_{\text {los }}^{\text {sat }}$ slope is even more increased.
In the MOND regime, the external field effect (EFE, e.g. Wu et al. 2007) would also change the predictions. This will modulate the actual force on the particle tracers, and on the velocity. Since there was still latitude for fitting the observations with MOND, we feel that it is still possible to consider it with other anisotropy parameters. However, we note that the external field effect is not even necessary, for reproducing the observed velocity dispersion slope.

\section{References}

Angus, G. W., Shan, H. Y., Zhao, H. S., \& Famaey, B. 2007a, ApJ, 654, L13 Angus, G. W., Famaey, B., Tiret, O., Combes, F., \& Zhao, H. S. 2007b, MNRAS, in press [arXiv:astro-ph/0709.1966]

Bosma, A. 1981, AJ, 86, 1825

de Blok, W. J. G. 2005, ApJ, 634, 227

Dekel, A., Stoehr, F., Mamon, G. A., et al. 2005, Nature, 437, 707

Douglas, N. G., Napolitano, N. R., Romanowsky, A. J., et al. 2007 [arXiv: astro-ph/0703047]

Famaey, B., Bruneton, J. P., \& Zhao, H. S. 2007, MNRAS, 377, L79

Gentile, G., Salucci, P., Klein, U., et al. 2004, MNRAS, 351, 903

Klypin, A., \& Prada, F. 2007 [arXiv:astro-ph/0706. 3554] (KP07)

Milgrom, M. 1983, ApJ, 270, 365

Navarro, J. F., \& Steinmetz, M. 2000, ApJ, 528, 607

Romanowsky, A. J., Douglas, N. G., Arnaboldi, M., et al. 2003, Science, 301, 1696

Rubin, V. C., Thonnard, N., \& Ford, W. K., Jr. 1980, ApJ, 238, 471

Sanders, R. H. 2003, MNRAS, 342, 901

Sanders, R. H., \& McGaugh, S. S. 2002, ARA\&A, 40, 263

Shapiro, K. L., Cappellari, M., de Zeeuw, T., et al. 2006, MNRAS, 370, 559

Verheijen, M. A., \& Sancisi, R. 2001, A\&A, 370, 765

Wu, X., Zhao, H. S., Famaey, B., et al. 2007, ApJ, 665, L101

Zhao, H. S. 2007, ApJL, in press [arXiv:astro-ph/0710.3616] 